\title{
Detection of IMBHs from microlensing in globular clusters
}

\author{
Margarita Safonova ${ }^{1}$ and Sohrab Rahvar ${ }^{2}$ \\ ${ }^{1}$ Indian Institute of Astrophysics, Koramangala, Bangalore, 560034, India \\ ${ }^{2}$ School of Physics, Institute for Theoretical Studies in Physics and Mathematics, \\ Farmanieh Building, Tehran, Iran \\ email: rita@iiap.res.in,rahvar@physics.ipm.ir
}

\begin{abstract}
Globular clusters have been long predicted to host intermediate-mass black holes (IMBHs) in their centres. The growing evidence that some/all Galactic globular clusters (GCs) could harbour middle range $\left(10^{2}-10^{4} M_{\odot}\right)$ black holes, just as galaxies do, stimulates the searches and the development of new methods for proving their existence. Here we propose another method of detection - the microlensing of the cluster stars by the central BH.
\end{abstract}

Keywords. Gravitational lensing, (Galaxy:) globular clusters: general

\section{Introduction}

The $\mathrm{BH}$ in a GC centre acts as a telescope with a lens of effective diameter $\sim 40 \mathrm{AU}$; we propose to consider the microlensing events expected when globular cluster star passes behind the central $\mathrm{BH}$, which acts as a lens. Globular clusters are very advantageous for microlensing search of IMBHs, since the location of both potential lens and sources and their relative motions are well constrained, removing the ambiguities usually presented in the microlensing events detected towards the bulge and the Magellanic Clouds. The Galaxy has $\sim 150$ globular clusters, each containing $\sim 10^{4}-10^{5}$ stars, and though a significant fraction of stars in the cores of GCs will be unresolved with the ground-based telescope, one can use the pixel lensing technique.

\section{Microlensing of stars in globular clusters}

As an example we show the details of calculations for the M15 globular cluster, a very promising cluster with the possible mass of the central BH $3.2 \times 10^{3} M_{\odot}$ (Gerssen et al. 2002). In a globular cluster, $D_{\mathrm{LS}} \ll D_{\mathrm{L}} \approx D_{\mathrm{S}}$, and the Einstein radius reduces to $R_{\mathrm{E}} \approx(2.8 \mathrm{AU})\left(M_{\mathrm{bh}} / M_{\odot}\right)^{1 / 2}\left(D_{\mathrm{LS}} / 1 \mathrm{Kpc}\right)^{1 / 2}$. The optical depth is the number of stars to be lensed by a central $\mathrm{BH}$, considering a cone with the cross-section $\pi R_{\mathrm{E}}^{2}(r), d N_{\text {event }}=$ $\left(\rho(r) / M_{*}\right) \pi R_{\mathrm{E}}^{2}(r) d r$. Using the Plummer density profile, we estimate the number of microlensing events for M15 as $N \approx 1.3 \times 10^{-4}$, which would mean that if we monitor the centres of about $10^{4}$ globular clusters we have a chance to see a microlensing event already in progress. To estimate the mean duration of events, we divide the mean Einstein radius by the central velocity dispersion $\sigma,\left\langle t_{\mathrm{E}}\right\rangle=\left\langle R_{\mathrm{E}}\right\rangle / \sigma$. The mean Einstein radius is $\left\langle R_{\mathrm{E}}\right\rangle=\left(\int_{0}^{\infty} P(r) R_{\mathrm{E}}(r) d r\right) /\left(\int_{0}^{\infty} P(r) d r\right)$, where $P(r)=d N_{\text {event }} / d r$ is the probability of a star being inside the Einstein ring of the central BH. For M15, $\left\langle R_{\mathrm{E}}\right\rangle=2.07 \mathrm{AU}$, and with $\sigma=12 \mathrm{~km} / \mathrm{sec}, t_{\mathrm{E}}=300$ days.

We calculate these quantities for a specific list of globular clusters, assuming the mass of the central BH as $10^{3} M_{\odot}$ for all candidates except M15 (see above) and G1 (Gebhardt et al. 2002), which is mainly motivated by the idea based on $M_{\mathrm{bh}}-\sigma$ correlation existing 
for galaxies. A possible source of contamination is the self-lensing of the GC objects by themselves. We estimate the self-lensing integrated optical depth to be $\tau \simeq 10^{-3}$, and the self-lensing rate for a typical GC as 0.05 events per one year of observation. The time scale of self-lensing is estimated to be less than a year.

\section{Choice of globular clusters and observational strategy}

We have included in our sample all Galactic proposed core-collapsed (CC) clusters (Trager et al. 1995), due to current belief that central BHs can only reside in centrally concentrated clusters (e.g., M15, a proto-typical CC cluster (Djorgovski \& King 1986; Lugger et al. 1997)). However, recently Baumgardt et al. (2004) claimed that, on the contrary, one has to look for medium-concentration King-profile clusters with nearly flat cores. We have included in our sample the candidates of Baumgardt as well.

Following the globular clusters classification scheme (Mackey \& van der Bergh 2005), we found that majority of the clusters candidates belong to the old halo/bulge-disk $(\mathrm{OH} / \mathrm{BD})$ group. We noticed that as far as cluster luminosity versus half-light radius is concerned, there is no considerable difference between the Baumgardt and CC sets. We noticed the clustering of our candidates in a small area of the plot, indicating the region where lie the clusters that are both tight and bright. It was noticed before (Gebhardt et al. 2002) that to the extent that a massive, bound cluster can be viewed as a 'mini-bulge', it may be that every dense stellar system (small or large) hosts a central black hole. We observe that, most probably, dense and high-luminosity clusters are better candidates for central BH search than diffuse and low-luminosity ones. Ideally, we would monitor all $\sim 150$ Galactic globular clusters. However, it just may be useless to look for central $\mathrm{BHs}$ in diffuse and faint clusters. Moreover, in CC clusters, if we take into account the mass segregation effect or more concentrated mass distribution law, a $r^{-7 / 4}$ profile, the lensing rate increases. For example, for nearby 47 Tuc, NGC 6397, and NGC 6752 it would nearly double.

\section{Conclusions}

Currently we are writing a proposal for a 2-m class telescope to look at the clusters for the central $\mathrm{BH}$ using the pixel lensing technique. We came to the tentative conclusion that some classes of GCs possess characteristics which indicate that they are more likely to be the ones to look for the central $\mathrm{BH}$. The $\mathrm{OH}, \mathrm{BD}$ and possible ex-spheroidals (like $\omega$ Cen) clusters are most likely ones. Since the average event duration is up to years, we can monitor clusters cores with the frequency once or twice a month, which gives the advantage of easily differentiating other possible sources of contamination, like selflensing or lensing of the stars belonging to the Galactic buldge or Magellanic Clouds backgrounds. Besides, no far-lying background stars can be detected within the highly crowded core radius, so any discovered event will be due to the stars within the cluster.

\section{References}

Gerssen, J., van der Marel, R. P., Gerbhardt, K., Guhathukurta, P., Peterson, R. C. \& Pryor, C. 2002, AJ, 124, 3270

Gerssen, J., van der Marel, R. P., Gerbhardt, K., Guhathukurta, P., Peterson, R. C. \& Pryor, C. 2003 , AJ 125, 376

Gerbhardt, K., Rich, R. M. \& Ho, L. C. 2002, ApJ 578, L41

Djorgovski, S. \& King, I. 1986, ApJ, 305, 61

Lugger, P. M., Cohn, H., Grindlay, J. E., Baylin, C. D. \& Hertz, P. 1987, ApJ, 320, 482

Trager, S. C., King, I. \& Djorgovski, S. 1995, AJ, 109, 218

Baumgardt, H., Makino, J. \& Hut, P. 2004, astro-ph/0410597

Mackey, A. D. \& van den Bergh, S. 2005, astro-ph/0504142 